\title{
Dynamics of Stress Activation in Repeated Stress Conditions in Experiment
}

\author{
DOI: $10.17691 / \mathrm{stm} 2019.11 .1 .18$
}

Received November 15, 2018

A.V. Bakhchina, PhD, Researcher, Laboratory of Psychophysiology named after V.B. Shvyrkov"; Leading Engineer, Laboratory for Behavior and Behavioral Ecology of Mammals²;

S.J. Laukka, Adjunct Professor, Researcher, Faculty of Education, Learning Research Laboratory (LearnLab) ${ }^{3}$;

S.B. Parin, DSc, Head of Interdepartmental Laboratory of Cognitive Psychophysiology";

V.V. Gavrilov, PhD, Senior Researcher, Laboratory of Psychophysiology named after V.B. Shvyrkov';

Senior Researcher, Laboratory of Neurocognitive Investigations of Individual Experience,

Institute of Experimental Psychology ${ }^{5}$

${ }^{1}$ Institute of Psychology, Russian Academy of Sciences, 13, Yaroslavskaya St., Moscow, 129366, Russia;

${ }^{2}$ A.N. Severtsov Institute of Ecology and Evolution, Russian Academy of Sciences, 33 Leninsky Prospect,

Moscow, 119071, Russia

3University of Oulu, 9 Yliopistokatu, P.O. Box 8000, Fl-90014, Finland;

${ }^{4}$ National Research Lobachevsky State University of Nizhny Novgorod, 23 Prospekt Gagarina, Nizhny Novgorod, 603950, Russia;

${ }^{5}$ Moscow State University of Psychology and Education, 29 Sretenka St., Moscow, 127051, Russia

The aim of the study was to analyze the dynamics of stress-specific indices of the body functional state, i.e. the level of catecholamines in blood plasma and spectral indices of heart rate variability (HRV) in repeated immobilization stress for identifying the effects of habituation.

Materials and Methods. The jugular veins of the rats (Long-Evans, $n=6)$ were catheterized before the experiment. For immobilization, the animals were fixed mildly in a special hammock for 30 min daily during 5 days. In the process of rat immobilization as well as for 30 min before and 60 min after immobilization under the normal conditions in the home cage, ECG was noninvasively recorded for a subsequent HRV analysis based on the spectral indices. On experimental days 1, 3, and 5, blood was collected 30 min before immobilization (stage 1), 5 min after it (stage 2), at the end of immobilization (stage 3), and $30 \mathrm{~min}$ after the end of the immobilization period (stage 4) to test the concentration of catecholamines in blood plasma.

Results. The effects of habituation to immobilization were found in the dynamics of catecholamine levels in blood plasma, heart rate, and HRV. The concentration of adrenaline and noradrenaline increased statistically significantly in the period of immobilization on the first experimental day and did not change significantly on the following days. Heart rate decreased significantly at stages 3 and 4 of the experiment (free behavior in the home cage after immobilization) on day 5 and remained at the initial high level on experimental days 1 and 3. The total power of the HRV spectrum statistically significantly decreased in the immobilization period on all experimental days. On day $1, \mathrm{HRV}$ remained reduced till stage 4 of the experiment. On days 3 and 5 , the total power of the HRV spectrum was restored after immobilization up to the initial level by stage 4 of the experiment.

Conclusion. Five-day exposure of the animals to the repeated immobilization stress in a special hammock designed for their fixation in electrophysiological experiments resulted in habituation which is demonstrated by the dynamics of catecholamine level in the blood and HRV. The data obtained showed that the effects of habituation are displayed not only by the reduction of the stress activation intensity but by the decrease of its duration as well.

Key words: repeated stress; immobilization; habituation; dynamics of catecholamines in the blood; heart rate variability spectrum.

\section{Introduction}

The interest to chronic stress is accounted for by the growing number of various diseases (especially cardiovascular) being their consequence [1]. The emphasis in chronic stress investigations is mainly focused on the dynamics of the individual's state and identification of its markers in order to improve the diagnosis [2]. Experimental procedures of forming chronic stress in individuals are developed for this purpose [1]. In this experimental procedures (e.g. the model of unpredictable chronic mild stress [3]) great attention is paid to the avoidance of habituation effects, i.e. adaptation of the individual to the initial stress conditions. However, the phenomenology of habituation and its dynamics is poorly studied just as well as all the factors determining the processes occurring during the repeated stress [4].

Corresponding author: Anastasia V. Bakhchina, e-mail: nastya18-90@mail.ru 
The habituation effects (or, vice versa, sensitization) during repeated stress are usually studied to test the models of stressors for the possibility of their multiple use in the experiments [5]. At the level of biochemical indices, no consistency of the effects in relation to habituation to the repeated stress-factor was found. Thus, the repetition of the common model of social stress (trier social stress test) showed that habituation effects are manifested by the dynamics of cortisol concentration (index of hypothalamus-pituitary axis) and alpha-amylase (index of alpha adrenoreceptor activation) in the saliva and is not observed in the dynamics of interleukin-6 concentration (index of inflammatory process intensity). At the physiological level, some characteristics of heart rate variability (HRV), i.e. mean value and R-R interval dispersion, are suggested as a possible way of evaluating the effects habituation in repeated cognitive stress [6]. Besides, the dynamics of these indices agreed with the behavioral effects of habituation. The intensity of habituation effects assessed by heart rate (HR) changes, systolic and diastolic pressure directly correlated with the indices of openness to a new experience evaluated on the basis of the questionnaire [7].

The available data urge forward the investigations of the dynamics and mechanisms of habituation to the repeated stressors as a simplest form of learning expressed in the reduction of stress-specific symptoms.

In addition to the applied aspect, studying habituation during repeated stress is also of great methodological importance. Usually, experimental protocols involving animals include the initial stage of adaptation (to the environment or to the necessary but unexplored action) to eliminate novelty and stress effects in the dynamics of the tested variables. Closer contact procedures (e.g. fixation of the animal in a device) require a longer period of adaptation. Additional application animal state control methods in the experiment will allow the researchers to eliminate reliably the possibility of stressogenic effects of the selected procedure. Absence of this possibility demands preliminary pilot series of experiments to identify the stage at which the realization of the experimental protocol causes no stress in the majority or all the animals. The most interesting investigation in this respect is the study of habituation during repeated immobilization stress since many experiments of electrophysiological investigations include animal mobility restrictions to various degree with nonpharmacological ways.

The aim of the study was to identify the dynamics of the individual's functioning state (according to stressspecific indices) under the repeated stress conditions in a model of immobilization stress.

\section{Materials and Methods}

The investigation was carried out on mature LongEvans male rats weighing on average $300 \mathrm{~g}$ and aged 19 months (range 6 to 24). The jugular vein was catheterized in 39 rats but due to some technical problems (catheter impassability), a full experimental series has been performed only on 6 animals.

The rats were housed under standard conditions of vivarium in individual cages with free access to standard rat feed and water. All manipulations were carried out in compliance with order No. $199 \mathrm{H}$ "On the Approval of the Rules of Good Laboratory Practice" (Russia, 2016), International Guiding Principles for Biomedical Research Involving Animals (CIOMS and ICLAS, 2012), and European Convention for the Protection of Vertebrate Animals used for Experimental and other Scientific Purposes (Strasbourg, 2006). The permission for experimental studies on animals was obtained from the Ethical Committee of the Institute of Psychology of the Russian Academy of Sciences.

Operation on jugular vein catheterization. Catheters represented silicon tubes $(2.5 \mathrm{~cm}$ long) and polyethylene tube (6 cm long). The sterile catheters were washed with the solution of $96 \%$ rubbing alcohol. Animals were anesthetized by intramuscular injection of Rometar $(10 \mathrm{mg} / \mathrm{kg})$ and Zoletil $(25 \mathrm{mg} / \mathrm{kg})$ solution. The operative field was made free from fur, shaved and treated with antiseptic. The incision was made through the skin along the oblique line connecting the upper third of the sternum and the internal third of the left or right clavicle in such a way that $2 / 3$ of the cut were below the clavicle. The incision was $1-1.5 \mathrm{~cm}$ long. Then the superficial fascia was dissected and the jugular vein separated by blunt dissection. Further, the ligatures were placed on the vein: permanent in the rostral direction and temporal in the caudal direction. Between them, the incision on the external wall of the jugular vein was performed. The obtained opening was widened with the branches of the microsurgical forceps and a silicon part of the catheter was inserted in the caudal direction to the depth of $2-3 \mathrm{~cm}$ weakening the caudal ligature. The introduced catheter was tested for the possibility to collect blood, filled with heparinized physiological solution (500 Units/ml, $80 \mu \mathrm{l})$, closed with a plug, and fixed with ligatures to the vein. It was thereafter subcutaneously exteriorized through the 3-4 mm incision between the scapulae and the wounds were sutured.

The catheter passability was checked daily for a week by collecting blood samples.

Experimental protocol. A week after the catheter placement, a 5-day experimental series with daily sessions including 4 stages each was performed (Figure 1). At stage 1 (30 $\mathrm{min})$, the rat was in its home cage under normal free conditions. At stage 2 (30 min), the rat was fixed in a special hammock for immobilization. At stages 3 and 4 (30 min each), the rat was again in its home cage under normal free conditions.

Non-invasive ECG was taken at each stage. On experimental days 1, 3, and 5 before stages 1, 3, 4 and at the fifth minute of stage $2,1 \mathrm{ml}$ of blood was collected through the catheter. The collected blood sample was 


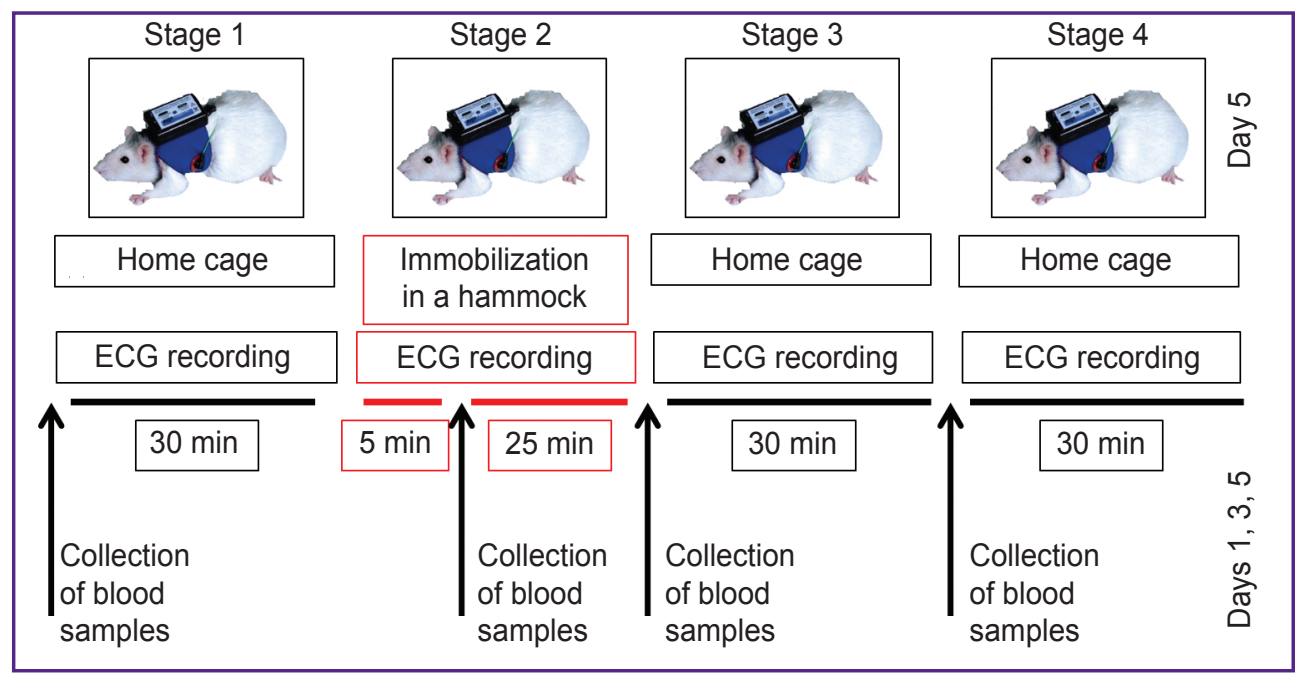

Figure 1. Design of experiment

centrifuged to separate plasma fraction and stored at $\mathrm{t}=18^{\circ} \mathrm{C}$.

HR recording. Physiobelt system $(35 \times 55 \times 15 \mathrm{~mm}$, $40 \mathrm{~g}, 500 \mathrm{~Hz}$ sampling rate) (Open Science, Russia) was used for non-invasive bipolar ECG recording. The system included a cuff for placement of the electrodes on the animal body and device attachments with a builtin amplifier, AD converter, and transmitter. Recording was supported by Physiobelt software (Neurobotics, Russia).

Identification of catecholamine concentration in blood plasma. The catecholamine level in blood plasma was assessed using isocratic chromatography system Gilson 407 (Gilson, France) and electrochemical LC-4C detector (BAS, USA) according to the protocol designed by Karimova et al. [8]. All reagents were chemically pure, analytical grade reagents.

Concentration of epinephrine and norepinephrine was determined in the blood plasma samples. These substances were chosen for the analysis because their concentration growth is a marker of acute stress stage I (the stage of sympathoadrenal system activation [9]).

Analysis of $H R$ variability. $R-R$ intervals (time intervals between the R-waves in the adjacent QRST complexes of a cardiogram) were isolated from the rat ECG by a threshold method using Spike 2.5 software. To isolate the heart rate sequences, $100 \mathrm{~s}$ ECG intervals without artefacts were selected. The obtained R-R intervals sequences were analyzed using Welch's method of periodograms for estimating power spectra having preliminarily interpolated them with a 50-s interval. For this purpose, Rhythm Service 1.2 software (Photon-test, Russia) was applied. As the result, the following parameters were calculated for each sequence of $\mathrm{R}-\mathrm{R}$ intervals: HR (per $\mathrm{min}$ ), average heart rate; TP $\left(\mathrm{ms}^{2}\right)$, total HRV spectrum power; LF $\left(\mathrm{ms}^{2}\right)$, spectrum power in the low-frequency region $(0.04-0.15 \mathrm{~Hz})$; $\mathrm{HF}\left(\mathrm{ms}^{2}\right)$, spectrum power in the high-frequency region
(0.15-0.60 Hz); LF/HF, the ratio of spectrum powers in the region of low and high frequencies (autonomic balance index). All described indices were calculated for each stage of the experiment as means of the values for all isolated $R-R$ intervals sequences with $100 \mathrm{~s}$ duration in this period.

Spectral indices were chosen to characterize HRV since the previous works $[10,11]$ showed that the reduction of the total power of the HRV spectrum and growth of the autonomic balance index are specific for the beginning of acute stress.

Statistical processing. For experimental days 1, 3 , and 5 , the dynamics of catecholamine level values in blood plasma and spectral HRV indices (stages 1 to 4) during the entire experiment were tested for the presence of significant changes using Friedman criterion [12]. Paired comparisons of the variables between the stages of the experiment and between the experimental days were performed using the Wilcoxon test [13]. Changes were considered as statistically significant at $\mathrm{p} \leq 0.05$.

\section{Results and Discussion}

The analysis of epinephrine concentration dynamics showed statistically significant changes $\left(\chi^{2} \quad(n=6 ; d f=3)=14.8 ; \quad p=0.002\right.$, Friedman criterion $)$ on day 1 of the experimental series. A statistically significant epinephrine growth was noted at stage 2 of the experiment (during animal immobilization) and its subsequent reduction by stage 3 and then by stage 4 (free behavior in the home cage) ( $\leq \leq 0.05$, Wilcoxon criterion). Similar changes were observed in the dynamics of norepinephrine $\left(\chi^{2}(n=6 ; d f=3)=8.8 ; p=0.03\right.$, Friedman criterion).

On day 3 of the experiment, there were no statistically significant changes in the dynamics of both epinephrine $\left(\chi^{2}=3.8 ; p=0.28\right.$, Friedman criterion) and norepinephrine 
$\left(\chi^{2}=1.0 ; p=0.80\right.$, Friedman criterion). Similarly, on day 5 of the experiment, there were no significant changes in the dynamics of epinephrine $\left(\chi^{2}=6.0 ; p=0.11\right.$, Friedman criterion) and norepinephrine $\left(\chi^{2}=2.4 ; p=0.49\right.$, Friedman criterion).

Epinephrine and norepinephrine values were statistically significantly higher at stage 2 on day 1 than at stage 2 on days 3 and 5 ( $p \leq 0.05$, Wilcoxon criterion for both variables). At stages 1,3 , and 4 , the values of epinephrine and norepinephrine did not differ statistically significantly on experimental days 1,3 , and 5 . The dynamics of epinephrine and norepinephrine during all experimental days is shown in detail in Figure 2.

Thus, according to the data of catecholamine concentration in blood plasma, stress activation was observed on day 1 in animals during immobilization in the experimental hammock which was manifested by a statistically significant growth of the epinephrine and norepinephrine level and the return to the initial level $30 \mathrm{~min}$ after the completion of mobilization. Effects of habituation to immobilization were registered in the dynamics of catecholamine concentration on days 3 and 5 , as the reliable changes of epinephrine and norepinephrine between the stages of the experiment were absent, and their significant reduction during immobilization in comparison with the first experimental day was also established. It may be supposed that habituation to immobilization in this case appeared not as reduction of stress activation but rather in the decrease of the time period in which it unfolded. If stress activation was triggered and finished in the first $5 \mathrm{~min}$ of immobilization, only initial level of epinephrine and norepinephrine have been found in the second blood plasma sample (which was collected 5 min after the beginning of experimental stage 2 ).

In the HR dynamics, no statistically significant changes were observed on experimental day $1\left(\chi^{2}=4.00\right.$; $p=0.261$, Friedman criterion $)$ and day $3\left(\chi^{2}=3.4 ; p=0.334\right.$, Friedman criterion). But reliable changes were detected on day $5 \quad\left(\chi^{2}=11.6 ; p=0.009\right.$, Friedman criterion): statistically significant HR reduction was observed from stage 2 (immobilization period) to stages 3 and 4 (free behavior in the home cage). The HR dynamics during all experimental days is shown in detail in Figure 3.

The HR level in the immobilization period did not differ during all experimental days and was about $460 \mathrm{bpm}$. The differences between the experimental days were observed at stage 3 and 4: on day 5 the HR at these stages was significantly lower than on days 1 and 3 ( $p \leq 0.05$ for both stages, Wilcoxon criterion).

Thus, the effects of habituation to the stressor were observed in the HR dynamics on experimental day 5 and consisted in the HR reduction upon the completion of immobilization. Absence of HR increase at the immobilization stage was likely to be connected with its initially high level at stage 1 on all days of the experiment which underlines once more a weak specificity of this index directly to stress.

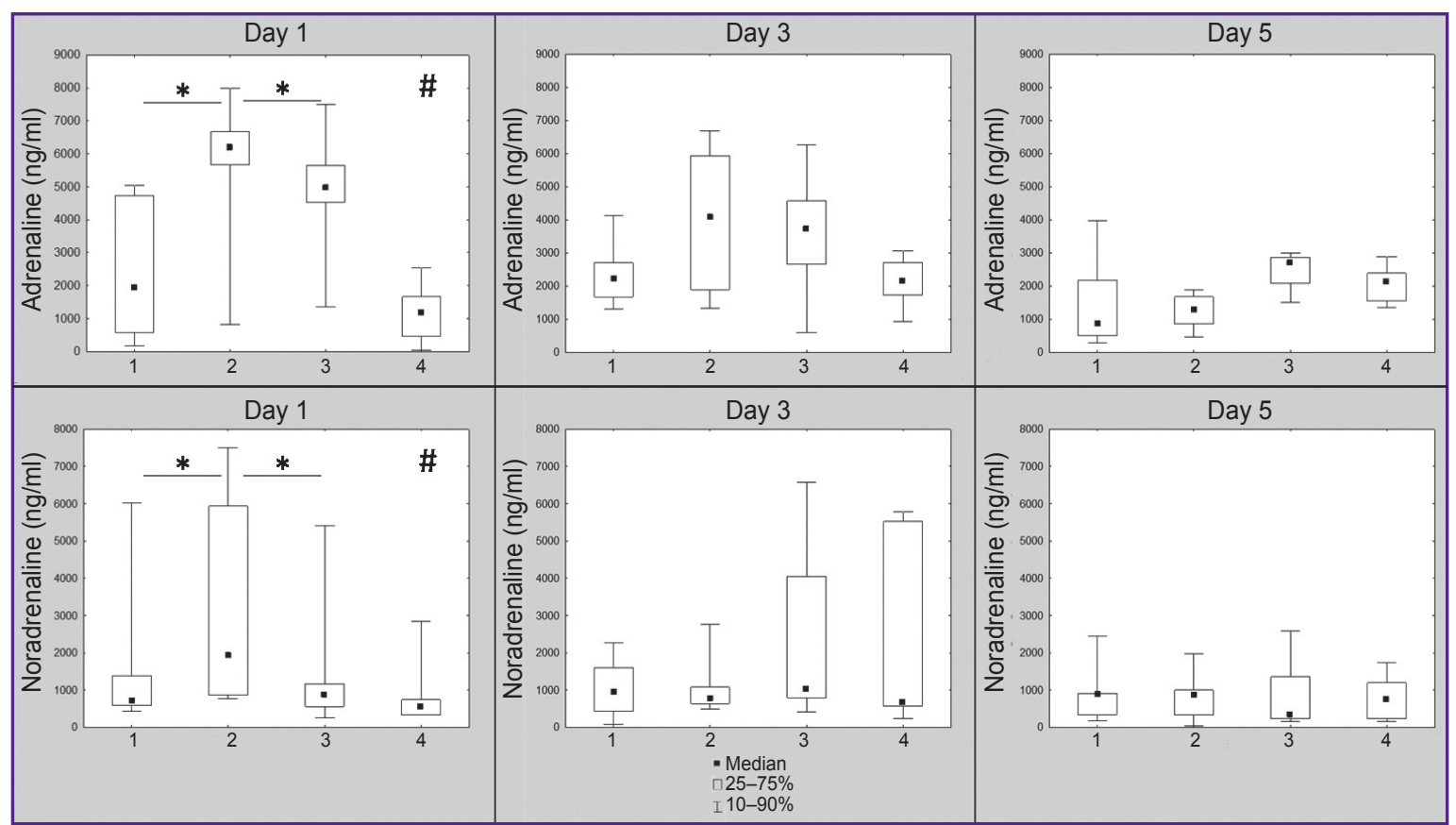

Figure 2. Dynamics of adrenaline and noradrenaline concentration $(\mathrm{ng} / \mathrm{ml})$ in the rat blood plasma in the experiment with immobilization (values of medians and percentiles of the distribution):

1 - at the beginning of the experimental stage 1 (30 min before immobilization); 2 - the fifth minute of stage 2 (immobilization in the hammock); 3 - at the beginning of stage 3 (immediately after the completion of immobilization); 4 - at the beginning of stage 4 (30 min after immobilization) on days 1,3 , and 5 of the experimental series; ${ }^{*} p \leq 0.05$, Wilcoxon criterion; ${ }^{\#} p \leq 0.05$, Friedman criterion 
In the dynamics of the total power of the $H R V$ spectrum, statistically significant changes were observed on experimental days $1\left(\chi^{2}=11.4 ; p=0.01\right.$, Friedman criterion), $3 \quad\left(\chi^{2}=9.0 ; \quad p=0.03\right.$, Friedman criterion), and 5 ( $\chi^{2}=10.6 ; p=0.02$, Friedman criterion). Its value statistically significantly decreased on all experimental days at stage 2 (immobilization period) relative to stage 1 (free behavior in the home cage) (in all cases $p \leq 0.05$, Wilcoxon criterion). On days 1 and 5 , the total power of the HRV spectrum statistically significantly increased at stage 3 (free behavior in the home cage) compared to stage 2 (immobilization) ( $p \leq 0.05$, Wilcoxon criterion). However, it statistically significantly decreased at stage 4 relative to stage 3 ( $p \leq 0.05$, Wilcoxon criterion) while it continued to increase on day 5 at stage 4 reaching the initial level. The comparison of the HRV values between the days of the experiment showed that they did not differ at stages 1, 2, and 3. And at the same time, the HRV values at stage 4 were statistically significantly higher on day 3 than 1 and on day 5 than 3 (see Figure 3).

Thus, on all days of the experiment, HRV assessed by the total spectral power of $R-R$ interval variations statistically significantly decreased in the immobilization period which is typical for stress activation. However, it should be noted that on experimental days 3 and 5 , in contrast to day 1, HRV has restored up to the initial level by stage 4 of the experiment. In other words, with the repetition of one and the same stress factor the activation, not changing by the degree of intensity, developed faster which might also be the effect of habituation as a quicker restoration after the stress impact.

In the dynamics of the autonomic balance index (LF/HF), there were no statistically significant

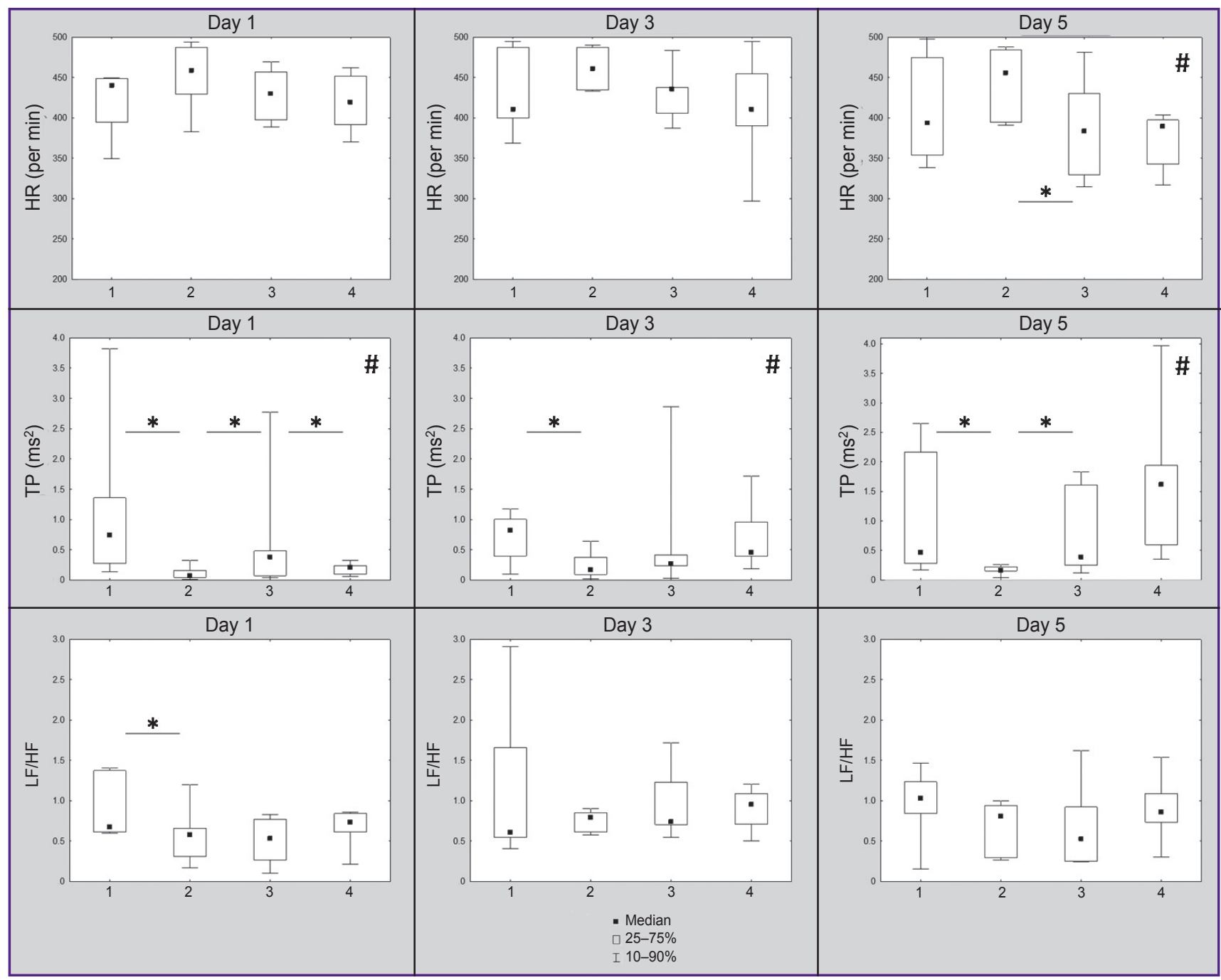

Figure 3. Dynamics of HR, total power of HRV spectrum (TP), and autonomic balance index (LF/HF) (values of medians and percentiles of the distribution) in the rats in the experiment with immobilization:

1 - during stage 1 of the experiment (30 min of free behavior in the home cage); 2 - during stage 2 (30 min of immobilization in the hammock); 3 - during stage 3 (30 min of free behavior in the home cage); 4 - during stage 4 (30 min of free behavior in the home cage) on days 1,3 , and 5 of the experimental series; ${ }^{*} p \leq 0.05$, Wilcoxon criterion; ${ }^{*} p \leq 0.05$, Friedman criterion 
changes on day $1\left(\chi^{2}=4.6 ; p=0.203\right.$, Friedman criterion), day $3\left(\chi^{2}=1.8 ; p=0.615\right.$, Friedman criterion), and day 5 $\left(\chi^{2}=0.6 ; p=0.896\right.$, Friedman criterion). On experimental day 1 , statistically significant reduction of this index was observed at stage 2 (immobilization) relative to stage 1 ( $p \leq 0.05$, Wilcoxon criterion). The dynamics of this index during all experimental days is shown in detail in Figure 3.

In the observed dynamics of the autonomic balance index, the increase typical for the stress activation was absent $[10,11]$. It can be explained by the specific selection of frequency range limits for the calculation of LF and HF values. In the given case, all computations were done according to the standard range limits (see section "Analysis of heart rate variability") which were accepted and used in the studies involving people. At the same time, there are data on species specificity of these indices [14], in particular, LF and HF ranges for the rats may be shifted to a higher frequency range: LF to 0.04 $1.0 \mathrm{~Hz}, \mathrm{HF}$ to $1.0-3.0 \mathrm{~Hz}$ [15]. It may be expected that the dynamics of this index will have some other structure if spectrum power ratios are recalculated in compliance with these ranges.

Thus, the results obtained showed that habituation of the rats exposed to the stress conditions had occurred by day 5 of daily repeated 30-min immobilization. Habituation effects are manifested both at the hormonal level (by the dynamics of catecholamine concentration) and at the level of the entire organism (by HRV indices).

A combined analysis of biochemical and electrophysiological data in the experiment enabled us to reveal an important aspect of habituation dynamics: it manifested itself not so much by the reduction of stress activation intensity as by the decrease of time during which it unfolds and, as a consequence, by the acceleration of parameter restoration to the initial background level. It is shown by the HRV data, i.e. the HRV reduction typical for stress was displayed in the period of immobilization even on day 5 and the values of the total power of HRV spectrum in the period of immobilization do not differ on day 1,3 , and 5 of the experiment.

Such dynamics of the heart rate characteristics gives grounds to suppose that when the time interval between the beginning of the stress factor and blood collection for biochemical analysis diminishes from 5 to $3 \mathrm{~min}$ and $1 \mathrm{~min}$, habituation according to the catecholamine dynamics will start to manifest itself later, i.e. after a greater number of stress factor repetitions. It is the methodological restrictions for the frequency of blood collection for the biochemical analysis (impossibility to increase time sampling for the obtained estimates) that may result in inconsistent effects of habituation in different biochemical stress markers [4, 16].

It is worth mentioning that continuous heart rate monitoring (from the beginning of stress activation to complete restoration of the initial level) may provide more ample information on the habituation dynamics.
In this case, it will be possible to accurately assess duration changes of stress activation in the repeated stress. And this, in its turn, will allow the verification of the hypothesis that habituation is the process of diminishing the time during which the activity of the stress-realizing body systems is unfolding.

\section{Conclusion}

The analysis of the dynamics of stress-specific indices of the body functional state, i.e. the level of catecholamines in blood plasma and spectral indices of heart rate variability under repeated immobilization stress, allowed us to conclude that effects of habituation are manifested not only by the reduction of stress activation intensity but by its duration as well.

Acknowledgement. The authors thank O.V. Kurskaya for teaching them catheterization of the rat jugular vein.

Study funding. The work was supported by the Russian Foundation of Basic Research, grant No.18313-20003 "Study of adaptation process dynamics in case of disagreement in solving socially significant tasks" (electrophysiological part), grant No.18-413520006_p_a "Development of Internet-oriented technology of the event-associated telemetry for screening, monitoring, and correction of stress-induced disorders in schoolchildren of Nizhny Novgorod", and grant No.0159-2019-0001 "Principles of systemogenesis and actualization of previously formed memory in the individual and collective behavior" (biochemical part) within the R\&D topic for 2019.

Conflicts of interest. The authors declare no conflicts of interest.

\section{References}

1. Golbidi S., Frisbee J.C., Laher I. Chronic stress impacts the cardiovascular system: animal models and clinical outcomes. Am J Physiol Heart Circ Physiol 2015; 308(12): H1476-H1498, https://doi.org/10.1152/ajpheart.00859.2014.

2. McEwen B.S. Neurobiological and systemic effects of chronic stress. Chronic Stress 2017; 1: 247054701769232 , https://doi.org/10.1177/2470547017692328.

3. Lucca G., Comim C.M., Valvassori S.S., Pereira J.G., Stertz L., Gavioli E.C., Kapczinski F., Quevedo J. Chronic mild stress paradigm reduces sweet food intake in rats without affecting brain derived neurotrophic factor protein levels. Curr Neurovasc Res 2008; 5(4): 207-213, https://doi.org/10.2174/1 56720208786413406.

4. Rohleder N., Janson J., Sturmbauer S. Mechanisms of habituation versus sensitization of inflammatory responses to repeated acute stress. Psychoneuroendocrinology 2017; 83: 74-75, https://doi.org/10.1016/j.psyneuen.2017.07.439.

5. Quaedflieg C.W.E.M., Meyer T., van Ruitenbeek P., Smeets T. Examining habituation and sensitization across repetitive laboratory stress inductions using the MAST. Psychoneuroendocrinology 2017; 77: 175-181, https://doi. org/10.1016/j.psyneuen.2016.12.009.

6. Feda D.M., Roemmich J.N. Effect of interpersonal and 
cognitive stressors on habituation and the utility of heart rate variability to measure habituation. Stress Health 2016; 32(4): 320-327, https://doi.org/10.1002/smi.2618.

7. Súilleabháin P.S.Ó., Howard S., Hughes B.M. Openness to experience and adapting to change: cardiovascular stress habituation to change in acute stress exposure. Psychophysiology 2018; 55(5): e13023, https://doi. org/10.1111/psyp.13023.

8. Karimova M.Kh., Kudrin V.S., Gaynetdinov R.R. Assessment of catecholamine content in the blood of practically healthy people. Klinicheskaia laboratornaia diagnostika 1993; 9(2): 33-35.

9. Parin S.B. Humans and animals in extreme situations: neurochemistry mechanisms, evolutionary aspect. Vestnik Novosibirskogo gosudarstvennogo universiteta. Seriya: Psikhologiya 2008; 2(2): 118-135.

10. Polevaya S.A., Runova E.V., Nekrasova M.M., Fedotova I.V., Bakhchina A.V., Kovalchuk A.V., Shishalov I.S., Parin S.B. Telemetry and information technologies in diagnosis of sportsmen functional state. Sovremennye tehnologii $v$ medicine 2012; (4): 94.

11. Bakhchina A.V. Dinamika vegetativnoy regulyatsii kardioritma pri kognitivnykh, emotsional'nykh i fizicheskikh nagruzkakh. Avtoref. dis. ... kand. psikhol. nauk [Dynamics of vegetative heart rate regulation during cognitive, emotional, and physical loads. PhD Thesis]. Moscow; 2014.

12. Hollander M., Wolfe D.A., Chicken E. Nonparametric statistical methods. John Wiley \& Sons, Inc.; 2015, https://doi. org/10.1002/9781119196037.

13. Wilcoxon F. Individual comparisons by ranking methods. Biometrics 1945; 1(6): 80-83, https://doi.org/10.2307/3001968.

14. von Borell E., Langbein J., Després G., Hansen S. Leterrier C., Marchant-Forde J., Marchant-Forde R., Minero M., Mohr E., Prunier A., Valance D., Veissier I. Heart rate variability as a measure of autonomic regulation of cardiac activity for assessing stress and welfare in farm animals - a review. Physiol Behav 2007; 92(3): 293-316, https://doi.org/10.1016/j. physbeh.2007.01.007.

15. Kuwahara M., Yayou K., Ishii K., Hashimoto S., Tsubone H., Sugano S. Power spectral analysis of heart rate variability as a new method for assessing autonomic activity in the rat. $J$ Electrocardiol 1994; 27(4): 333-337, https://doi. org/10.1016/s0022-0736(05)80272-9.

16. Pulopulos M.M., Vanderhasselt M.-A., De Raedt R. Association between changes in heart rate variability during the anticipation of a stressful situation and the stress-induced cortisol response. Psychoneuroendocrinology 2018; 94: 6371, https://doi.org/10.1016/j.psyneuen.2018.05.004. 